\title{
The learning to criterion of a serial list with and without mnemonic instructions
}

\section{P. S. DELIN, University of Adelaide, South Australia 5001}

A group of Ss with instructions to use a mnemonic technique and a group with standard serial anticipation instructions learned a list of English nouns to a criterion of one error-free anticipation. Mnemonic $S$ s made fewer errors and took fewer trials to criterion. They also took longer to respond, and made a higher proportion of their errors in the form of omissions. The learning of a second list, and backward recall, were also investigated.

A number of researchers (e.g., Wallace, Turner, \& Perkins, 1957; Smith \& Noble, 1965; Bugelski, Kidd, \& Segmen, 1968) have reported some degree of facilitation of learning when Ss were instructed to use a mnemonic technique involving imagery. In none of these studies, however, was mnemonic learning compared with nonmnemonic both on the kind of material for which the mnemonics were designed and, at the same time, under normal serial anticipation conditions. The fact that mnemonic Ss make fewer errors when a single trial is given does not entail their making fewer errors when a number of trials are given. It could be that control Ss are better able to take advantage of the later trials. Part of the object of this study was to check this possibility.

An implicit claim of those who promulgate mnemonics is that the person who uses them does something that the rote learner does not do: that he interposes between stimulus and response a complex and, presumably, time consuming, mediating process. It would be consistent with this claim that mnemonic Ss should take longer to respond than control Ss. It was therefore decided that a response latency measure should be taken.

Users of mnemonics report that when they forget their mnemonic images they find it difficult to make any response to the stimulus item. The writer, in another study (Delin, 1968), found differences in predominant error type associated with differences in subjective reports on explicit mediation. A corollary of the report that loss of the mnemonic leads to inability to respond is the hypothesis that mnemonic Ss should make more of their errors in the form of omissions than do control Ss, and it was decided that this hypothesis should be explicitly tested.
Since this could be done without interference with the main objects of the study, it was decided to collect some data relevant to two other issues. Firstly, it was decided that some Ss should be asked to learn a second list. Wallace et al (1957) reported a considerable learning-to-learn effect with mnemonic Ss, resulting, they thought, from increased efficiency in the application of the mnemonic technique. They did not, however, use control Ss. Wood (1966) did find positive nonspecific transfer to be greater for a group using a mnemonic aid than for a control group, but he was concerned with a type of mnemonic very different from that used by Wallace et al, or by the present writer.

Secondly, it was decided that some Ss would be asked to recall the experimental list in the reverse direction. Mnemonists claim that they are able to recall a list backwards with the same facility with which they can recall it forwards. Although a number of authors (e.g., Young, Patterson, \& Benson, 1963) have investigated backward serial recall, finding little transfer from forward to backward learning, they have not used either the type of material or the length of presentation time involved in the present study.

METHOD
The material used in the study needed to be suitable for the use of mnemonic imagery. The most suitable material consists of familiar object names. There being little reason for imposing precise limitations of meaningfulness or frequency, the following procedure was adopted. A large number of people were individually approached and asked to name the first object that sprang to mind. The resulting collection of object names, when duplicated responses and two-word names had been excluded, constituted the pool from which two 16-item lists (L1 and L2) were randomly drawn.

The Ss, 64 first-year psychology students, were randomly assigned to mnemonic and control groups. From each of the resulting groups of $32 \mathrm{Ss}, 12$ were randomly selected to learn a second list and 10 to relearn the first list in the reverse direction.

All Ss learned L1 which was presented on a memory drum set for $7 \mathrm{sec}$ presentation time. There was a $4 \mathrm{sec}$ interval after each item had been presented, during which $S s$ were expected, on all trials after the first, to attempt to anticipate the next item. Intertrial interval was $30 \mathrm{sec}$. Learning was carried to a criterion of one error-free trial. The interitem interval was provided to enable the $E$ to record response time in such a way that it would be distinct from recognition time. A pen-recorder was at tached to the memory drum, and recorded when each item was presented and the start of the anticipation interval. The $E$ could place another mark on the tape by pressing a button when the $S$ made a response.

The mnemonic Ss were instructed to try to connect each item to the preceding one by making a vivid and active mental image containing the two items. They were told that the image should be as bizarre or fantastic as possible. Even if the two items went together in some quite natural way they were to eschew this mundane connection in favor of an unnatural one. They were to avoid connecting the images together in a string, but were to make an independent image for each pair. It was further suggested that if they made an incorrect anticipation they should spend the remainder of the presentation time "strengthening" the image they had previously made. The control Ss were given standard serial anticipation instructions.

Twelve Ss in each group learned L2 under the same conditions and instructions as $\mathrm{L} 1$. Ten other Ss from each group relearned $\mathrm{LI}$ in the reverse direction after reaching criterion in the forward direction. The length of delay before relearning and the level of distraction during the delay period experienced by these backward relearning Ss were varied as the experiment proceeded.

\section{RESULTS}

As can be seen from Table 1, mnemonic Ss performed better than control Ss in terms of both trials and errors before criterion on L1. The differences between means, tested with a Mann-Whitney $U$ test, were both highly significant. For errors $\mathrm{p}$ was smaller

Table 1

Means, SDs, and Medians for Trials and Errors Data

\begin{tabular}{llrrrr}
\hline & & \multicolumn{2}{c}{ Mnemonic Ss } & \multicolumn{2}{c}{ Control Ss } \\
\cline { 3 - 6 } & & Trials & Errors & Trials & Errors \\
\hline List 1 & Mean & 3.27 & 12.00 & 5.08 & 26.46 \\
Each $\mathrm{n}=32$ & SD & 1.64 & 5.31 & 1.43 & 8.63 \\
& Median & 3.00 & 9.00 & 5.00 & 28.00 \\
List 2 & Mean & 2.13 & 3.50 & 3.33 & 10.08 \\
Each $\mathrm{n}=12$ & SD & 1.12 & 2.43 & 2.67 & 3.88 \\
& Median & 2.00 & 3.00 & 3.50 & 8.00 \\
\hline
\end{tabular}


than $.002(z=3.11)$, and for trials it was smaller than $.005(z=2.83)$.

The latency measure was scored as the number of $1 / 4 \mathrm{sec}$ before a response was made, thus producing a 17-point scale. This measure was used because it was felt that the way in which the response latency was recorded did not justify a direct use of the times recorded on the tape. For the first anticipation trial, counting only those items on which a response was made, the weighted mean for mnemonic Ss was 11.4, while that for control Ss was 8.5. This difference was significant at the .01 level on a test $(t=2.94)$. On later trials these latency scores tended both to reduce and to converge, so that the differences were not significant after the first trial.

For both groups the errors made were classified as omissions ( $O$ ), or as commissions (C). Extralist intrusions were rare (only seven were made altogether) so that $\mathrm{C}$ errors were almost exclusively intralist intrusions. For mnemonic Ss the means were $8.23(\mathrm{O})$ and $3.75(\mathrm{C})$, while for control Ss they were $15.63(\mathrm{O})$ and $10.79(\mathrm{C})$. In order to assess the significance of the intergroup difference in predominant error type, the 0 score of each $S$ was expressed as a proportion of his total score, and a Mann-Whitney $U$ test was used to compare the two sets of proportions. This proved significant at the .05 level $(\mathrm{z}=2.21)$.

As can be seen from Table 1 the results of the $12 \mathrm{Ss}$ in each group who learned L2 were similar to those obtained on the first list, in that mnemonic Ss again made fewer errors and took fewer trials to reach criterion. In spite of the reduced numbers of Ss, both of these differences were significant on Mann-Whitney $U$ tests $[\mathrm{p}<.001, \mathrm{U}=15$ (errors) and $\mathrm{p}<.01, \mathrm{U}=25$ (trials)]. A savings score was computed for each $\mathrm{S}$ by dividing his error score on L2 by his error score on $\mathrm{L} 1$, subtracting the resulting proportion from 1.00 , and expressing the result as a percentage. For the mnemonic Ss the mean savings score was $72.6 \%$, while the equivalent mean for the control $\mathrm{Ss}$ was 57.3\%. The difference just reached significance $(\mathrm{p}<.05, \mathrm{U}=36)$ on a Mann-Whitney $\mathrm{U}$ test. No equivalent analysis was carried out for the trials data, as it was evident that this would not reach significance. An analysis of the $\mathrm{O}$ and $\mathrm{C}$ errors, carried out in the same way as for L1, again found that the mnemonic Ss made a significantly $(\mathrm{p}<.01$, $U=24$ ) higher proportion of $O$ errors.

The first 3 of the 10 Ss from each group who relearned $L 1$ in the reverse direction did so immediately after reaching criterion in the forward direction. Since only one error was made (by a control S), a further three Ss from each group were delayed for $20 \mathrm{~min}$ after learning $L 1$ before relearning it backwards. Again only one $S$, this time a mnemonic one, made errors (he made two). Finally, four Ss from each group relearned L1 backwards after a delay period of $30 \mathrm{~min}$ during which they worked at mathematical problems. This time one control and two mnemonic Ss made one error each. These data, then, gave little indication that backward relearning is a difficult task for either mnemonic or control Ss given the type of material and mode of presentation under consideration.

\section{DISCUSSION}

The main results of this study make it clear that the advantages conferred by mnemonic instructions persist when the material is presented more than once. The results seem even more impressive when it is considered that the mnemonic Ss in this study had neither a demonstration nor an opportunity to practice it before the experimental task. Subjective reports and image descriptions collected after the formal sessions suggested that most mnemonic $S$ s had failed to make images for some of the items, and that few of the images created had been very bizarre. Conversely, a number of the control Ss reported using mnemonic techniques not very different from the one the mnemonic Ss had been instructed to use. These comments suggest that the observed mnemonic/control differences underestimate those in principle attainable.

Many of the mnemonic Ss felt that what they were doing was very different from trying to learn a list "by heart," while many of the control Ss described themselves as "just trying to recite the words off." It became clear that the Ss, rightly or wrongly, distinguished two kinds of learning, which might loosely be called "rote" and "nonrote."

The findings on latency are not inconsistent with these subjective reports. Thus, it might be suggested that mnemonic Ss initially took longer to respond because they had to recall and decode their mnemonic, whereas control Ss had only to call for the next item. The convergence of the latencies in later trials is consistent with the findings of Adams \& McIntyre (1967) that natural language mediators tend to drop out with successive recall trials. Similarly, the tendency of mnemonic Ss to make more of their errors in the form of omissions is consistent with the claim of professional mnemonists that if they cannot remember their mnemonic they do not remember anything at all. It could be that the control Ss, when they call for an item out of the memory store, are not expecting it to come with a mnemonic certificate of identity, and so are more likely to make an intrusion error.

The results on the second list, while not conclusive, are consistent with the view that mnemonic Ss improve rapidly in their use of the mnemonic technique. It is true that the difference in transfer did not appear in the data on trials to criterion, but the mnemonic Ss had taken so few trials to reach criterion on the first list that they had very little room for improvement in this regard. Part of the general improvement in performance from first to second list could be accounted for in terms of accidental differences in list difficulty, but this would not account for the intergroup differences, which were particularly impressive in view of the small sample-size.

The findings on backward relearning, although based on even smaller numbers of Ss, were both clear and surprising. It had been expected that the control Ss at least would find the task difficult. The fact that neither group did so suggests that we are here dealing with a situation radically different from that dealt with by Young et al (1963). Specifically, it suggests that with this type of material and mode of presentation the functional stimulus tends to be item-based rather than order-based.

\section{REFERENCES}

ADAMS, J. A., \& McINTYRE, J. S. Natural language mediation and all-or-none learning. Canadian Journal of Psychology, 1967, 21 , 436-449.

BUGELSKI, B. R., KIDD, E., \& SEGMEN, J. Image as a mediator in one-trial paired-associate learning. Journal of Experimental Psychology, $1968,76,69-73$.

DELIN, P. S. The effects of high and low meaningfulness and interitem association upon the level of difficulty of a serial task. Psychonomic Science, 1968, 12, 71-72.

SMITH, K., \& NOBLE, C. E. Effects of a mnemonic technique applied to verbal learning and memory. Perceptual \& Motor Skills, 1965, 21, 123-134.

WALLACE, W. H., TURNER, S. H., \& PERKINS, C. C. Preliminary studies of human information storage. D.A. Project No. 3-99-12-023. S.C. Project No. 1220. University of Pennsylvania, 1957.

WOOD, G. Mnemonic systems in recall. Unpublished PhD dissertation, Northwestern University, 1966.

YOUNG, R.K., PATTERSON, H., \& BENSON, W. N. Backward serial learning. Joumal of Verbal Learning \& Verbal Behavior, 1963, 1, 335-338. 